\title{
IDENTIFICATION OF TIME VARYING \\ CARDIAC DISEASE STATE USING A MINIMAL CARDIAC MODEL WITH REFLEX ACTIONS.
}

\author{
Christopher E. Hann*, Steen Andreassen**, \\ Bram W. Smith ${ }^{* *}$, Geoffrey M. Shaw ${ }^{* * *}$, \\ J. Geoffrey Chase*, Per L. Jensen ${ }^{* * * *}$ \\ * Centre for Bioengineering, University of Canterbury, \\ Christchurch, New Zealand \\ ** Centre for Model-based Medical Decision Support, \\ Aalborg University, Aalborg, Denmark \\ *** Christchurch Hospital Department of Intensive Care \\ Medicine,Christchurch, New Zealand \\ **** Department of Cardiology, Aalborg Hospital, Denmark
}

\begin{abstract}
A minimal cardiac model that accurately captures the essential cardiovascular system dynamics has been developed. Standard parameter identification methods for this model are highly non-linear and non-convex, hindering clinical application, given the limited measurements available in an intensive care unit. This paper presents an integral based identification method that transforms the problem into a linear, convex problem. Five common disease states including four fundamental types of shock, are identified to within $10 \%$ without false identification. Clinically, it enables medical staff to rapidly obtain a patient specific model to assist in diagnosis and therapy selection.
\end{abstract}

Keywords: Biomedical Systems, Physiological Models, Integrals, Parameter Identification, Diagnosis

\section{INTRODUCTION}

Heart disease is difficult to diagnose and treat due to often confusing clinical data that does not directly point to the problem. The body's natural reflex actions can hide a particular disease state. Hence, medical staff often rely on experience and intuition.

A minimal cardiac model has been developed that captures the major dynamics of the cardiovascular system (CVS) (Smith et al., 2004). To assist medical staff in diagnosis and treatment, a fast accurate patient-specific parameter identification method is required. The model is minimal in the sense that a minimum number of variables and equations are used to describe the common heart disease states.

However, the model does not lend itself to a convex identification problem (Smith, 2004). Thus, potentially false solutions could be found. Furthermore, to implement common non-linear regression identification methods (Carson and Cobelli, 2001) requires many computationally expensive model simulations (Smith, 2004). Hence, computational intensity severely limits the number of optimization iterations available to find a solution in a clinically useful time period. 
In this paper, the model is reformulated in terms of integrals of measured data so that patient specific identification is linear and convex and does not require the differential equations of the model to be solved. Five common disease states, including the four fundamental types of shock, are simulated from onset. Each disease state is then identified in the presence of $10 \%$ uniformly distributed noise. The body's reflex actions to keep the pressure in the aorta stable are included. The model parameters are identified to capture the dynamic progression of each disease from the initial healthy state using the pressure waveforms through the aorta and pulmonary artery, the flows through the ventricles and their maximum and minimum volumes. More importantly, all of these measurements are available in critical care using Swan-Ganz catheters or ultra-sound.

\section{METHODOLOGY}

\subsection{Cardiac Model}

The full model consists of six elastic chambers as shown in Figure 1.

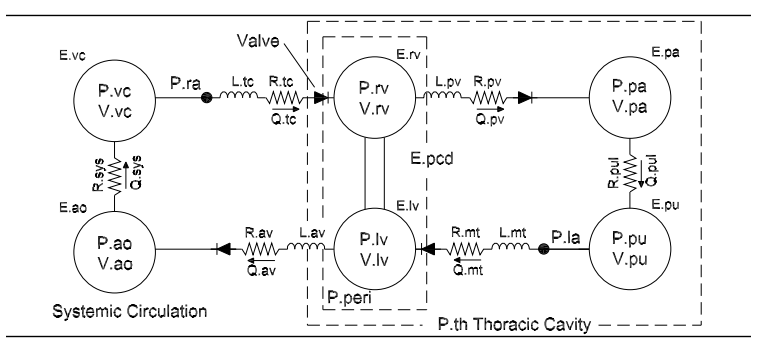

Fig. 1. The full six chamber cardio-vascular system model

For simplicity, only the differential equations associated with the left ventricle are shown, see (Smith et al., 2004) for a description of the full model. For computational efficiency, the differential equations for the left ventricle are defined (Hann et al., 2005a):

$$
\begin{aligned}
L_{\mathrm{av}} \dot{Q}_{\mathrm{av}}=H\left(H\left(P_{\mathrm{lv}}-P_{\mathrm{ao}}\right)\right. & \left.+H\left(Q_{\mathrm{av}}\right)-0.5\right)\left(P_{\mathrm{lv}}\right. \\
& \left.-P_{\mathrm{ao}}-R_{\mathrm{av}} Q_{\mathrm{av}}\right)
\end{aligned}
$$

$$
\begin{aligned}
L_{\mathrm{mt}} \dot{Q}_{\mathrm{mt}}=H\left(H\left(P_{\mathrm{pu}}-P_{\mathrm{lv}}\right)\right. & \left.+H\left(Q_{\mathrm{mt}}\right)-0.5\right)\left(P_{\mathrm{pu}}\right. \\
& \left.-P_{\mathrm{lv}}-R_{\mathrm{mt}} Q_{\mathrm{mt}}\right)
\end{aligned}
$$

$$
\begin{aligned}
\dot{V}_{\mathrm{lv}} & =Q_{\mathrm{mt}}-Q_{\mathrm{av}} \\
\dot{V}_{\mathrm{ao}} & =Q_{\mathrm{av}}-Q_{\mathrm{sys}} \\
P_{\mathrm{ao}} & =E_{\mathrm{ao}} V_{\mathrm{ao}} \\
Q_{\mathrm{sys}} & =\frac{P_{\mathrm{ao}}-P_{\mathrm{vc}}}{R_{\mathrm{sys}}}
\end{aligned}
$$

$$
\begin{aligned}
& P_{\mathrm{lv}}= e(t) E_{\mathrm{es}, \mathrm{lvf}}\left(V_{\mathrm{lv}}-V_{\mathrm{spt}}\right) \\
&+(1-e(t)) P_{0, \mathrm{lvf}}\left(e^{\lambda_{\mathrm{lvf}}\left(V_{\mathrm{lv}}-V_{\mathrm{spt}}\right)}-1\right) \\
&+P_{\mathrm{peri}} \\
& e(t)= e^{-80(t-\tau / 2)^{2}} \\
& \tau \equiv \text { period of heart beat } \\
& H(K(t))=0, \quad K(t) \leq 0 \\
&=1, \quad K(t)>0
\end{aligned}
$$

where $H$ in Equation (11) is the Heaviside function, $Q_{\mathrm{av}}$ and $Q_{\mathrm{mt}}$ are the flows through the aortic valve and mitral valve of the left ventricle, $P_{\mathrm{ao}}$, $P_{\mathrm{pu}}$ and $P_{\mathrm{lv}}$ are the pressures in the aorta, pulmonary vein and left ventricle, $V_{\mathrm{lv}}$ is the volume in the left ventricle, $V_{\mathrm{spt}}$ is the septum volume and $P_{\text {peri }}$ is the pressure in the pericardium (Smith et al., 2004). The Heaviside formulation of Equations (1) and (2) provides an open on pressure close on flow valve law (Smith et al., 2004) such that:

$$
\begin{aligned}
L_{\mathrm{av}} \dot{Q}_{\mathrm{av}}=P_{\mathrm{lv}}-P_{\mathrm{ao}}-R_{\mathrm{av}} Q_{\mathrm{av}}, & Q_{\mathrm{mt}}=0 \\
L_{\mathrm{mt}} \dot{Q}_{\mathrm{mt}}=P_{\mathrm{pu}}-P_{\mathrm{lv}}-R_{\mathrm{mt}} Q_{\mathrm{mt}}, & Q_{\mathrm{av}}=0
\end{aligned}
$$

where Equation (12) holds during ejection stage and Equation (13) holds during filling.

\subsection{Reflex actions}

The effect of heart disease and shock on the CVS can be significantly altered by the body's natural reflex response that attempts to maintain enough blood pressure and flow to sustain life. The affect of reflex actions can thus often mask the underlying problem and must be accounted for in the CVS model.

Reflex actions included are divided into four groups: vaso-constriction, venous constriction, increased heart rate (HR) and increased ventricular contractility (Burkhoff and Tyberg, 1993). Their activation is assumed to be proportional to the drop in the average pressure in the aorta $\left(P_{\text {ao }}\right)$. The proportionality constants are estimated based on clinically observed CVS hemodynamic responses reported in the literature (Braunwald, 1997; Despopoulos and Silbernagl, 2001; Parrillo and Bone, 1995).

Specifically, vaso-constriction is simulated in the model based on increasing the systemic resistance $R_{\text {sys }}$ by $35 \%$ for a drop in average $P_{\text {ao }}$ from $100 \mathrm{mmHg}$ to $80 \mathrm{mmHg}$. Similarly, venous constriction, HR and ventricular contractility are increased based on increasing the venous dead space $V_{\mathrm{d}, \mathrm{vc}}, \mathrm{HR}$ and the left and right ventricle free wall contractilities $E_{\text {es,lvf }}$ and $E_{\text {es,rvf }}$ by $67 \%, 80$ to 120 beats per minute and $35 \%$ respectively for a drop in average $P_{\text {ao }}$ to $80 \mathrm{mmHg}$. For example Figure 2 shows how $V_{\mathrm{d} \text {,vc }}$ is varied as a function of 
$\Delta P_{\text {ao }}$ which is the amount the average $P_{\text {ao }}$ differs from $100 \mathrm{mmHg}$. In the model simulations, reflex actions are applied every heart beat.

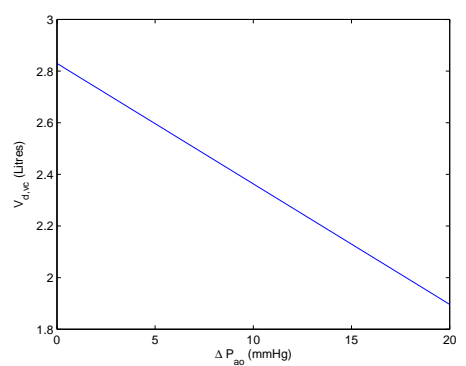

Fig. 2. Varying $V_{\mathrm{d}, \mathrm{vc}}$ as a function of $\Delta P_{\mathrm{ao}}$

\subsection{Integral parameter identification}

Consider the left ventricle Equations (12) and (13), and Equations (3)-(6), and assume that $Q_{\mathrm{av}}$, $Q_{\mathrm{mt}}, P_{\mathrm{ao}}, V_{\mathrm{lv}, \max }, V_{\mathrm{lv}, \text { min }}, \lambda_{\mathrm{lvf}}, V_{\mathrm{spt}}$ and $P_{\text {peri }}$ are either measured or estimated. In this case, the unknown input patient specific parameters are $R_{\mathrm{av}}, R_{\mathrm{mt}}, R_{\mathrm{sys}}, E_{\mathrm{es}, \text { lvf }}$ and $P_{0, \mathrm{lvf}}$. Integrating Equation (3) from $t_{e}$ to $t$ during ejection and from $t_{f}$ to $t$ during filling gives an expression for $V_{\mathrm{lv}}(t)$ defined:

$$
\begin{aligned}
V_{\mathrm{lv}}(t) & =V_{\mathrm{lv}}\left(t_{e}\right)-\int_{t_{e}}^{t} Q_{\mathrm{av}} d t, \quad t_{e} \leq t \leq t_{f} \\
& =V_{\mathrm{lv}}\left(t_{f}\right)+\int_{t_{f}}^{t} Q_{\mathrm{mt}} d t, \quad t_{f} \leq t \leq t_{e}+\tau
\end{aligned}
$$

where,

$$
\begin{aligned}
t_{e} & \equiv \text { beginning of ejection stage } \\
t_{f} & \equiv \text { beginning of filling stage } \\
V_{\mathrm{lv}}\left(t_{e}\right) & =V_{\mathrm{lv}, \max } \equiv \text { max left ventricle volume } \\
V_{\mathrm{lv}}\left(t_{f}\right) & =V_{\mathrm{lv}, \min } \equiv \text { min left ventricle volume }
\end{aligned}
$$

Integrating Equation (4) from 0 to $t$, solving for $V_{\text {ao }}(t)$ and then using Equations (5) and (6) yields:

$$
\begin{aligned}
P_{\mathrm{ao}} & =E_{\mathrm{ao}}\left(V_{\mathrm{ao}}(0)+\int_{0}^{t} Q_{\mathrm{av}}-\frac{1}{R_{\mathrm{sys}}} \int_{0}^{t} P_{\mathrm{ao}}\right. \\
& \left.-\frac{1}{R_{\mathrm{sys}}} \int_{0}^{t} P_{\mathrm{vc}}\right)
\end{aligned}
$$

which assuming that $P_{\mathrm{vc}}=P_{\mathrm{vc} 0}$ is an unknown constant can be written as

$$
P_{\mathrm{ao}}=V_{\mathrm{ao} 0}+E_{\mathrm{ao}} \int_{0}^{t} Q_{\mathrm{av}}+A \int_{0}^{t} P_{\mathrm{ao}}+B t
$$

where,

$$
V_{\mathrm{ao} 0}=E_{\mathrm{ao}} V_{\mathrm{ao}}(0), \quad A=-\frac{E_{\mathrm{ao}}}{R_{\mathrm{sys}}}, \quad B=-\frac{E_{\mathrm{ao}} P_{\mathrm{vc} 0}}{R_{\mathrm{sys}}}
$$

are treated as unknown constants.

Thus, the best linear least squares fit of Equation (21) to the measured pressure waveform $P_{\text {ao }}$ over one particular heart beat will determine $E_{\text {ao }}$ and $R_{\text {sys }}$ over that heart beat. Similarly, given an approximation to $V_{\text {spt }}$ and $P_{\text {peri }}$ in Equation (8), Equations (12) and (13) can be integrated across the filling and ejection stages respectively and a linear optimization can be set up to determine $R_{\mathrm{av}}, R_{\mathrm{mt}}, E_{\mathrm{es}, \mathrm{lvf}}$ and $P_{0, \mathrm{lvf}}$ (Hann et al., 2004). The right ventricle can be treated similarly.

The end result is that given the pressure waveforms through the aorta and pulmonary artery, the flows into and out of the left and right ventricles and their maximum and minimum volumes, a system of linear equations can be defined:

$$
\begin{aligned}
& A \underline{\beta}=b \\
& \underline{\beta}=\left[\underline{\alpha}, P_{\mathrm{ao} 0}, P_{\mathrm{pu} 0}, P_{\mathrm{pa} 0}, P_{\mathrm{vc} 0}\right] \\
& \underline{\alpha}=\left[L_{\mathrm{av}}, L_{\mathrm{mt}}, L_{\mathrm{tc}}, L_{\mathrm{pv}}, E_{\mathrm{es}, l \mathrm{vf}}, P_{0, \mathrm{lvf}}, E_{\mathrm{es}, \mathrm{rvf}}\right. \\
& \quad P_{0, \mathrm{rvf}}, R_{\mathrm{av}}, R_{\mathrm{mt}}, R_{\mathrm{tc}}, R_{\mathrm{pv}}, E_{\mathrm{vc}}, E_{\mathrm{pu}}, E_{\mathrm{ao}}, E_{\mathrm{pa}}, \\
& \left.\quad R_{\mathrm{sys}}, R_{\mathrm{pul}}\right]
\end{aligned}
$$

where $A$ is an $N \times 18$ matrix, $N>>18$ is the number of chosen integration periods over which the parameters are constant, $b$ is an $N \times 1$ vector, $\underline{\alpha}$ are the patient specific parameters and the initial conditions, $P_{\mathrm{ao} 0}, P_{\mathrm{pu} 0}, P_{\mathrm{pa} 0}$ and $P_{\mathrm{vc} 0}$ are treated as extra unknown variables. Equation (23) can then be solved by linear least squares to uniquely determine $\underline{\alpha}$.

\subsection{Simulating Disease States}

The disease states that are simulated are Pericardial Tamponade, Pulmonary Embolism, Cardiogenic Shock, Septic shock and Hypovolemic Shock. Pericardial tamponade is an excessive build up of fluid in the pericardium limiting ventricular expansion. It is simulated by reducing the pericardium dead-space volume $V_{0, \text { pcd }}$ by 20 $\mathrm{ml}$ every 10 heart beats for a total of 50 heart beats. Pulmonary Embolism is caused by a blood clot obstructing the pulmonary circulation and is simulated by increasing the resistance $R_{\text {pul }}$ by $20 \%$ every 10 heart beats for 50 heart beats.

Cardiogenic shock occurs when the heart is unable to pump a sufficient amount of blood to provide oxygen to the tissues and myocardium (Braunwald, 1997; Parrillo and Bone, 1995). Lack of oxygen supply to the myocardium causes further depression of cardiac function by decreas- 
ing ventricular contractilities and increasing diastolic elastance (Hollenberg et al., 1999). Hence, a patient beginning to suffer from left ventricular infarction due to a coronary artery becoming blocked is simulated from an initial healthy state. The left ventricle contractility is reduced in piecewise constant steps to $50 \%$ of normal and diastolic elastance is increased in piecewise constant steps to a factor of 2.5 due to ischemia.

Septic shock is a common type of distributive shock that is characterized by a drop in systemic vascular resistance. The beginning stages of septic shock are simulated by decreasing the systemic resistance $R_{\text {sys }}$ by $6 \%$ every 10 heart beats for 50 heart beats, giving a net $30 \%$ decrease in $R_{\text {sys }}$.

Finally, hypovolemic shock is caused by a severe drop in total blood volume, significantly reducing stressed blood volume. It is due to dehydration, hemorrhage or other fluid losses from the body. It is simulated by reducing the total volume of the blood by $250 \mathrm{ml}$ every 10 heart beats for 50 heart beats totalling a 1 litre blood loss.

\section{RESULTS}

A healthy human is simulated first, producing the results shown in Table 1.

Table 1. Pressure and volume outputs for a healthy human.

\begin{tabular}{ll}
\hline Volume in left ventricle & $111.7 / 45.7 \mathrm{ml}$ \\
Volume in right ventricle & $112.2 / 46.1 \mathrm{ml}$ \\
Cardiac output & $5.3 \mathrm{~L} / \mathrm{min}$ \\
Max $P_{\mathrm{lv}}$ & $119.2 \mathrm{mmHg}$ \\
Max $P_{\mathrm{rv}}$ & $26.2 \mathrm{mmHg}$ \\
Pressure in aorta & $116.6 / 79.1 \mathrm{mmHg}$ \\
Pressure in pulmonary artery & $25.7 / 7.8 \mathrm{mmHg}$ \\
Average pressure in pulmonary vein & $2.0 \mathrm{mmHg}$ \\
Average pressure in vena cava & $2.0 \mathrm{mmHg}$ \\
\hline
\end{tabular}

Pericardial tamponade is then simulated producing a significant rise in the pressure in the pulmonary vein to $7.9 \mathrm{mmHg}$, a reduction in cardiac output to $4.1 \mathrm{~L} / / \mathrm{min}$ and a reduction in mean arterial pressure to $88.0 \mathrm{mmHg}$. This result captures the physiological trends (Parrillo and Bone, 1995; Braunwald, 1997). Figure 3 shows the expected pressure change in the aorta.

All the other disease state simulations also produce trends in agreement with known physiological response. Model responses include: significantly increased of pulmonary artery pressure for Pulmonary Embolism; decreased mean arterial pressure, decreased cardiac output and elevated pulmonary vein pressure for Cardiogenic Shock; falling blood pressures and increased cardiac output for Septic Shock; and falling blood pressures and cardiac output for Hypovolemic Shock. Tables 2-6 show the full results as "true values". Trend magnitudes are also in good agreement with limited clinical data. Note that for hypovolemic shock, accounting for reflex actions that change the venous dead space volume every heart beat, the loss of 1 litre of blood amounts to a loss of $600 \mathrm{ml}$ of stressed blood volume. In this case, the stressed blood volume is the quantity which is identified.

The output pressures through the aorta and pulmonary artery and the flows through the chambers for all disease states are then discretized by sampling every 0.005 s and $10 \%$ random uniformly distributed noise is added using a random number generator in Matlab, analogous to measured data. Figure 4 shows the non-smooth pressure in the aorta for Pericardial Tamponade for one heart beat after random noise is added.

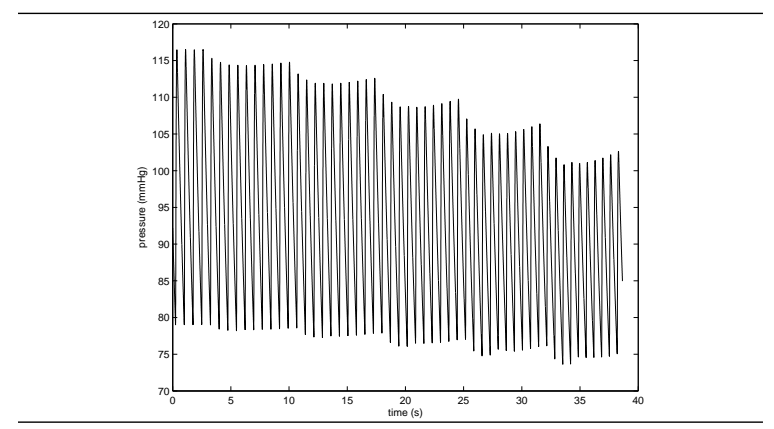

Fig. 3. The pressure though the aorta during dynamic simulation of the disease state pericardial tamponade from onset.

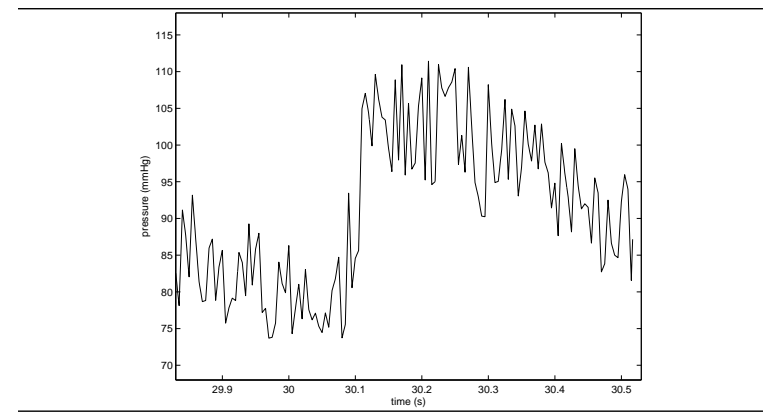

Fig. 4. The pressure through the aorta for one heart beat after $10 \%$ random uniformly distributed noise is added.

The integral method is then applied to identify each disease state as it progresses from an initial healthy state in the presence of $10 \%$ uniformly distributed noise. Note that one extra parameter, the pericardium dead-space volume $V_{0, p c d}$, is included in the optimization for all disease states. This parameter is embedded non-linearly in the matrix $A$ of Equation (23), so it is optimized by a depth first search to minimize $\| A\left(V_{0, \mathrm{pcd}}\right) \beta-$ $b \|_{2}$. Each evaluation of $\left\|A\left(V_{0, \mathrm{pcd}}\right) \underline{\beta}-b\right\|_{2}$ involves solving Equation (23) by linear least squares. For Hypovolemic shock the stressed volume was also included in the optimization, which changes when $V_{\text {d,vc }}$ changes due to reflex action. The stressed volume was calculated from the sum of the initial 
volumes in the left and right ventricles, which are known from Equation (14), and an analogous equation for the right ventricle, and the sum of $V_{\mathrm{ao}}(0), V_{\mathrm{pu}}(0), V_{\mathrm{pa}}(0)$ and $V_{\mathrm{vc}}(0)$, which can be computed using the values of $P_{\mathrm{ao} 0}, P_{\mathrm{pu} 0}, P_{\mathrm{pa} 0}$ and $P_{\mathrm{vc} 0}$ from Equation (24) and formulae similar to $V_{\text {aoo }}$ given in Equation (22).

Table 2 shows the identification results for Pericardial Tamponade. The particular disease state values of $V_{0, p c d}$ are all identified within $3 \%$ and all other parameters are identified within a mean error of 10\%. Tables 3-6 show the results for the other disease states. The total mean over all disease states and values identified was $4.0 \%$, ranging from $0-10 \%$. Note that when inertances are not included, as shown by the numbers in brackets, the mean and standard deviation values are significantly reduced. The reason for this last result is that inertances can change quite significantly (approximately 10-30\%) without having a major affect on dynamics. As they represent the inertia of blood volumes, they are difficult to measure and not well defined (Smith, 2004). The total mean error in all parameters across all disease states including inertances was $7.3 \%$ and without inertances was $3.6 \%$.

\section{DISCUSSION AND CONCLUSIONS}

Five disease states, Pericardial Tamponade, Pulmonary Embolism, Cardiogenic Shock, Septic Shock and Hypovolemic shock are dynamically simulated from an initial healthy state with reflex actions applied every heart beat. Results match reported physiological trends. An integral based optimization successively identified each disease as it developed within mean errors ranging from $0-10 \%$ in the presence of significant simulated measurement noise. The integrals are effectively a low pass filter so the method is independent to the chosen structure of noise. The integral method also turns a previously non-linear and non-convex optimization into a linear optimization with minimal computation required.

Note that the robustness of the method under noise refers only to uncertainty in measurement. It is assumed in this case that the model captures the fundamental underlying dynamics. If some dynamics are not captured it will show up as an error in fitting. However, there is the option of either adding further dynamics to the model or making some of the parameters in $\underline{\alpha}$ of Equation (25) time varying similar to (Hann et al., 2005b). The former risks adding physiological dynamics that do not "exist" outside the noisy measurements, the latter would only increase the number of unknown parameters in Equation (23), so there would still be a unique least squares solution.
A major advantage of the integral method is that it allows significant flexibility in adding further complexity to the model, such as atrial dynamics without significantly affecting computational time. Overall, the speed and accuracy of the integral based identification method demonstrates the potential of using this model in a clinical setting, to assist medical staff in diagnosis and therapy in clinically useful time (3-5 minutes) on a standard desktop computer.

\section{REFERENCES}

Braunwald, E. (1997). Heart Disease, A text book of cardiovascular medicine, 5th edition. W.B. Saunders Company, Philadelphia.

Burkhoff, D. and J. V. Tyberg (1993). Why does pulmonary venous pressure rise after onset of lv dysfunction: a theoretical analysis. $A m ~ J$ Physiol 265, H1819-H1828.

Carson, E. and C Cobelli (2001). Modelling Methodology for Physiology and Medicine. Academic Press.

Despopoulos, A. and S. Silbernagl (2001). Color atlas of physiology (5th Ed). New York: Thieme.

Hann, C. E., J. G. Chase and G. M. Shaw (2005a). Efficient implementation of non-linear valve law and ventricular interaction dynamics in the minimal cardiac model. Computer Methods and Programs in Biomedicine. In Press.

Hann, C.E., J.G. Chase, G.M. Shaw and B.W. Smith (2004). Identification of patient specific parameters for a minimal cardiac model. Proc 26 th International Conf of IEEE Engineering in Med and Biology Society (EMBS 2004), San Francisco, CA, Sept 1-5 pp. 813816.

Hann, C.E., J.G. Chase, J. Lin, T. Lotz, C.V. Doran and G.M. Shaw (2005b). Integral-based parameter identification for long-term dynamic verification of a glucose-insulin system model. Computer Methods and Programs in Biomedicine 77(3), 259-270.

Hollenberg, S. M., C. J. Kavinsky and J. Parrillo (1999). Cardiogenic shock. Ann Intern Med 131, 47-59.

Parrillo, J.E. and R.C. Bone (1995). Critical Care medicine, Principles of diagnosis and management. Mosby, St. Louis, Missouri.

Smith, B.W. (2004). Minimal Haemodynamic Modelling of the Heart and Circulation for Clinical Application. PhD thesis. University of Canterbury.

Smith, B.W., J.G. Chase, R. I. Nokes, G.M. Shaw and G. Wake (2004). Minimal haemodynamic system model including ventricular interaction and valve dynamics. Med. Eng. Phys 26(2), 131-139. 
Table 2. Pericardial tamponade (determining $V_{0, \mathrm{pcd}}$ ). The Numbers in the brackets are without including errors in the inertances.

\begin{tabular}{llllll}
\hline & $\begin{array}{l}\text { True value }(\mathrm{ml}) \\
\text { (to the nearest } \mathrm{ml})\end{array}$ & $\begin{array}{l}\text { Optimized } \\
\text { value }\end{array}$ & $\begin{array}{l}\text { Error }(\%) \\
V_{0, \mathrm{pcd}}\end{array}$ & $\begin{array}{l}\text { Mean } \\
\text { (all parameters) }\end{array}$ & Standard Deviation \\
\hline First change & 180 & 176 & 2.22 & $5.60(3.42)$ & $8.89(4.56)$ \\
Second change & 160 & 158 & 1.25 & $8.03(4.25)$ & $9.32(3.68)$ \\
Third change & 140 & 138 & 1.43 & $6.84(3.20)$ & $9.29(4.61)$ \\
Fourth change & 120 & 117 & 2.50 & $8.95(5.37)$ & $10.46(4.92)$ \\
Fifth change & 100 & 100 & 0 & $8.58(3.96)$ & $10.17(6.73)$ \\
\hline
\end{tabular}

Table 3. Pulmonary Embolism (determining $R_{\text {pul }}$ ).

\begin{tabular}{llllll}
\hline & $\begin{array}{l}\text { True value } \\
\left(\mathrm{mmHg} s \mathrm{ml}^{-1}\right)\end{array}$ & $\begin{array}{l}\text { Optimized } \\
\text { value }\end{array}$ & $\begin{array}{l}\text { Error (\%) } \\
R_{\text {pul }}\end{array}$ & $\begin{array}{l}\text { Mean } \\
\text { (all parameters) }\end{array}$ & Standard Deviation \\
\hline First change & 0.1862 & 0.1907 & 2.41 & $7.93(2.83)$ & $10.01(4.17)$ \\
Second change & 0.2173 & 0.2050 & 5.67 & $6.32(3.47)$ & $7.62(2.87)$ \\
Third change & 0.2483 & 0.2694 & 8.50 & $7.26(2.78)$ & $9.50(4.10)$ \\
Fourth change & 0.2794 & 0.2721 & 2.60 & $8.90(3.95)$ & $10.84(5.01)$ \\
Fifth change & 0.3104 & 0.2962 & 4.59 & $7.64(2.73)$ & $9.61(4.74)$ \\
\hline
\end{tabular}

Table 4. Cardiogenic Shock (determining $\left.\left[E_{\mathrm{es}, \mathrm{lvf}}, P_{0, \mathrm{lvf}}\right]\right)$.

\begin{tabular}{llllll}
\hline & $\begin{array}{l}\text { True values } \\
\left(\left[\mathrm{mmHg} \mathrm{ml}{ }^{-1}, \mathrm{mmHg}\right]\right)\end{array}$ & $\begin{array}{l}\text { Optimized } \\
\text { values }\end{array}$ & $\begin{array}{l}\text { Error }(\%) \\
{\left[E_{\mathrm{es}, \mathrm{lvf}}, P_{0, \mathrm{lvf}}\right]}\end{array}$ & $\begin{array}{l}\text { Mean } \\
(\text { all parameters })\end{array}$ & $\begin{array}{l}\text { Standard } \\
\text { Deviation }\end{array}$ \\
\hline First change & {$[2.5918,0.1564]$} & {$[2.6148,0.1478]$} & {$[0.89,5.49]$} & $8.41(5.42)$ & $9.15(5.12)$ \\
Second change & {$[2.3038,0.1925]$} & {$[2.2960,0.1840]$} & {$[0.34,4.39]$} & $5.35(2.85)$ & $9.22(2.38)$ \\
Third change & {$[2.0159,0.2286]$} & {$[2.0246,0.2102]$} & {$[0.43,8.03]$} & $6.27(3.43)$ & $9.04(3.06)$ \\
Fourth change & {$[1.7279,0.2647]$} & {$[1.7023,0.2386]$} & {$[1.48,9.85]$} & $6.73(3.93)$ & $9.65(5.28)$ \\
Fifth change & {$[1.4399,0.3008]$} & {$[1.4331,0.2725]$} & {$[0.47,9.39]$} & $8.41(5.42)$ & $9.15(5.12)$ \\
\hline
\end{tabular}

Table 5. Septic Shock (determining $R_{\text {sys }}$ ).

\begin{tabular}{llllll}
\hline & $\begin{array}{l}\text { True value } \\
\left(\mathrm{mmHg} s \mathrm{ml}^{-1}\right)\end{array}$ & $\begin{array}{l}\text { Optimized } \\
\text { value }\end{array}$ & $\begin{array}{l}\text { Error (\%) } \\
R_{\text {sys }}\end{array}$ & $\begin{array}{l}\text { Mean } \\
\text { (all parameters) }\end{array}$ & Standard Deviation \\
\hline First change & 1.0236 & 1.0278 & 0.41 & $6.64(3.55)$ & $9.02(4.06)$ \\
Second change & 0.9582 & 0.9714 & 1.37 & $6.17(2.23)$ & $8.43(4.10)$ \\
Third change & 0.8929 & 0.8596 & 3.73 & $7.37(3.75)$ & $10.09(4.94)$ \\
Fourth change & 0.8276 & 0.8316 & 0.49 & $6.43(3.15)$ & $8.45(3.35)$ \\
Fifth change & 0.7622 & 0.7993 & 4.86 & $8.58(4.03)$ & $10.28(5.04)$ \\
\hline
\end{tabular}

Table 6. Hypovolemic Shock (determining stressed blood volume).

\begin{tabular}{llllll}
\hline & $\begin{array}{l}\text { True value } \\
(\mathrm{ml})\end{array}$ & $\begin{array}{l}\text { Optimized } \\
\text { value }\end{array}$ & $\begin{array}{l}\text { Error }(\%) \\
\text { Stressed volume }\end{array}$ & $\begin{array}{l}\text { Mean } \\
\text { (all parameters) }\end{array}$ & Standard Deviation \\
\hline First change & 1299.9 & 1206.5 & 7.18 & $7.86(4.32)$ & $10.41(4.52)$ \\
Second change & 1177.3 & 1103.7 & 6.26 & $4.66(1.73)$ & $7.20(2.56)$ \\
Third change & 1063.1 & 953.8 & 10.28 & $7.34(3.14)$ & $10.86(4.47)$ \\
Fourth change & 967.8 & 1018.9 & 5.28 & $5.88(2.02)$ & $7.52(3.68)$ \\
Fifth change & 928.5 & 853.4 & 8.10 & $9.40(3.98)$ & $9.58(3.68)$ \\
\hline
\end{tabular}

